\title{
GCU
}

Glasgow Caledonian

University

University for the Common Good

\section{Statistical analysis for brain EIT images}

Zhang, Yan; Passmore, Peter; Bayford, Richard

DOI:

10.1109/MEDIVIS.2005.17

Publication date:

2005

Document Version

Author accepted manuscript

Link to publication in ResearchOnline

Citation for published version (Harvard):

Zhang, Y, Passmore, P \& Bayford, R 2005, 'Statistical analysis for brain EIT images', Paper presented at Medical Information Visualisation - Biomedical Visualisation, 2005. (MediVis 2005) Third International

COnference on, London, United Kingdom, 5/07/05 - 7/07/05 pp. 60-67. https://doi.org/10.1109/MEDIVIS.2005.17

\section{General rights}

Copyright and moral rights for the publications made accessible in the public portal are retained by the authors and/or other copyright owners and it is a condition of accessing publications that users recognise and abide by the legal requirements associated with these rights.

Take down policy

If you believe that this document breaches copyright please view our takedown policy at https://edshare.gcu.ac.uk/id/eprint/5179 for details of how to contact us. 


\title{
Statistical Analysis for Brain EIT Images Using SPM
}

\author{
Yan Zhang ${ }^{1}$, Peter J. Passmore ${ }^{1}$, Rebecca J. Yerworth ${ }^{2}$, Richard H. Bayford ${ }^{2,3}$ \\ ${ }^{1}$ School of Computer Science, Middlesex University, London, UK, N17 8HR \\ ${ }^{2}$ Department of Clinical Neurophysiology and Medical Physics, UCL, London, UK, WIN 8AA86 \\ ${ }^{3}$ School of Health and Social Sciences, Middlesex University, London, UK, EN3 4SA \\ y.zhang@mdx.ac.uk
}

\begin{abstract}
Electrical Impedance Tomography (EIT) is a relatively new medical imaging method, which is based on the physiological property that different tissues have different impedances. EIT imaging of brain provides neuroimages by detecting functional impedance changes in the brain caused by cell swelling, blood volume and flow increase, and neuronal depolarisation. These changes are very subtle, therefore imaging analysis is usually needed to enhance the images. In this paper, statistical processing is adopted to analyse brain EIT images. The feasibility of using SPM (Statistical Parametric Mapping), a popular statistical software package used for neuroimages obtained by SPECT/PET or FMRI, to analyse simulated brain EIT images is studied. A scheme of utilizing SPM to interpret brain EIT data is presented. The experimental results suggest that it is reasonable to process brain EIT images with SPM.
\end{abstract}

\section{Introduction}

Electrical Impedance Tomography (EIT) is a recently developed tomography imaging method. The physiological basis of EIT is that different tissues have different impedances. EIT imaging exploits this property (i.e. impedance) by injecting a small current through sensors encompassing the area to be imaged. Besides EIT, so far there are no other medical imaging methods that reveal this information. EIT imaging of the brain provides neuroimages by detecting functional impedance changes in the brain caused by three main mechanisms: a) cells outrun their energy supply and so swell, which cause the tissue impedance rises by tens of percent over minutes [8]; b) blood volume and flow increase during normal functional activity, which increases the local brain impedance by a few percent over minutes [12]; c) during neuronal depolarisation, ion channels open in the dendritic membrane causing its resistance to decrease a few percent over tens of milliseconds [10].

Currently, EIT is not in routine biomedical use for any purpose, but studies have applied EIT to measure functional brain activity during: stroke [8], cortical spreading depression [2], visual evoked responses [12], and epilepsy [11]. EIT imaging is cheap, safe, and portable. Compared with other functional imaging approaches, EIT has higher temporal resolution than SPECT (Single Photon Emission Computed Tomography), PET (Positron Emission Tomography), and fMRI (Functional Magnetic Resonance Imaging), and similar spatial-temporal resolution as EEG (Electroencephalogram) and MEG (Magneto encephalography). Considering the non-uniqueness in the reconstruction of EEG and MEG, EIT has the advantage to be uniquely reconstructed [9].

During functional imaging of the brain, or neuroimaging, the induced changes of regional neural activity are very subtle. Image analysis is usually needed to enhance the images. Because statistical analysis methods can deal with random events and processes, they are ideal to be used to model the mechanisms that cause image degradation and enable a more reliable detection of changes in the brain.

As a pioneer, Duffy and his colleagues [4] proposed significance probability mapping and proved it is useful for the analysis of topographic maps of brain electrical activity imaged by EEG. In the late 1980s and early 1990s, the research of functional brain image was mainly done with PET. Since the middle of 1990s, most researchers moved their attention to fMRI. Now, several statistical packages are available for the analysis of fMRI, such as, AFNI (Analysis of Functional Neuroimages) [3], MEDx [Sensor Systems Inc., 1996], and SPM (Statistical Parametric Mapping) [6], SPM is the most prevalent approach to characterizing functional anatomy and disease-related changes in the brain. Currently, the SPM method and software are applied to the analysis of SPECT, PET 
and $\mathrm{fMRI}$ images, and there are some research plans to apply SPM method to process brain EEG images.

Current research interests in EIT are mainly focused on improvement of reconstruction algorithms and development of imaging instrumentation. There is less research effort given to EIT image analysis. In this paper, statistical analysis is adopted to process brain EIT images. The feasibility of using SPM to interpret brain EIT images is studied.

\section{SPM}

Statistical Parametric Mapping (SPM) refers to the construction of spatially extended statistical processes to test hypotheses about regionally specific effects [6].

\subsection{Overview of SPM}

With SPM, the analysis of functional neuroimaging data involves many steps that can be broadly divided into three:

- Spatial processing

- Estimating the parameters of a statistical model

- Making inferences about those parameter estimates with their associated statistics.

The first step, spatial processing, aims to reduce artifactual variance components in the voxel timeseries that are induced by movement or shape differences among a series of scans. The next two steps are parameter estimation based on GLM (General Linear Model), and hypothesis inference based on GRF (Gaussian Random Field).

In SPM, the same model form is applied to every voxel simultaneously, with different parameters for each voxel. The observed values of a voxel under the experiment conditions are considered as a response variable $^{x_{i j}}$ ( $i=1,2, \cdots I$ index the observation (scan); $j=1,2, \cdots J$ index the voxel), and experimental conditions are described with a series of explanatory variables $g_{i k}$ under which the observation $i$ was made. The GLM is used to express the response variables in terms of a linear combination of explanatory variables plus an error term:

$$
x_{i j}=g_{i 1} \beta_{i j}+g_{i 2} \beta_{2 j}+\cdots+g_{i K} \beta_{K j}+\varepsilon_{i j}
$$

Where $\beta_{k j}$ are $\mathrm{K}$ unknown parameters for each voxel $j ; \varepsilon_{i j}$ are the errors.

Equation (1) can be written in matrix form:

$$
X=G \underline{\beta}+\underline{\varepsilon}
$$

Where $X$ has one column for each voxel $j$ and one raw for each scan $i$;

$G$ is comprised of the coefficients $g_{i k}$ and is called the Design Matrix, the Design Matrix has one row for each scan and one column for every effect in the model;

$\underline{\beta}$ is the parameter matrix where $\underline{\beta}_{j}$ is a column vector of parameters for voxel $j$;

$\underline{\mathcal{E}}$ is a matrix of error terms.

The parameter matrix $\underline{\beta}$ in the linear model can be achieved by Least Squares Estimates:

$$
\hat{\beta}=\left(G^{T} G\right)^{-1} G^{-1} X
$$

From this it follows that linear compounds of the parameters can be assessed by comparing with a $t$ (or $F$ ) distribution. As a result of the parameter estimation, a three dimension statistical image or 'map' formed of thousands of correlated $t$ or $F$ statistics is obtained. This map is named statistical parametric map.

The obtained statistical image contains a great number of voxels so it is not directly interpretable. It is clear that performing a statistical test at each and every voxel engenders an enormous false-positive rate using conventional and unadjusted thresholds to declare an activation as significant. So, a null hypothesis that no activation accounts for the experimental conditions appearing at each voxel is made. Under this null hypothesis, the statistical image can be transformed and considered as a Gaussian Random Field. In the Gaussian random field, a series of statistical variables based on different inference level can be constructed. By testing the statistical probability of these variables, the 'unlikeness' of these statistical variables is interpreted as regionally specific effects, attributable to the experimental context.

\subsection{Underling Assumptions of SPM}

To some extent, SPM is a combination of GLM and GRF. The underlying principle of GLM is a hypothesis:

- The error term in the GLM is normally distributed. Similarly, there are two assumptions underlying the use GRF correctly:

- The error fields (but not necessarily the data) are a reasonable lattice approximation to an underlying random field with a multivariate Gaussian distribution.

- These fields are continuous, with a twicedifferentiable autocorrelation function.

The only way in which these two assumptions can be violated is if:

- The data are not smoothed (with or without subsampling of the data to preserve resolution), violating the reasonable lattice assumption.

- The statistical model is mis-specified so that the errors are not normally distributed. [5] 


\section{Methods}

\subsection{Scheme of Processing EIT Data in SPM}

According to the criteria mentioned in section 2.2, the following conclusion can be made: in order to investigate if it is reasonable to use SPM to process an image data, two examinations must be done. The first one is to test if the data are smoothed; the second one is to correctly specify the statistical model and then test if the error term in the GLM is normally distributed.

For EIT data, we argue that it is smoothed. Unlike some other tomography approaches, initial reconstruction result of 3D EIT imaging is a 3D volume dataset instead of a stack of 2D slices. In order to get the sliced dataset, initial reconstruction data is interpolated and rasterized, which makes the final reconstruction data smoothed.

Friston [7] pointed out that the data obtained with image devices are uniform to Gaussian distribution. Even if the original physiological measurements were not Gaussian, after the explicit and implicit convolutions during reconstruction and smoothing of image as a pre-processing step, they will be (nearly). On the other hand, due to the Central Limit Theorem, which means if an error such as $\varepsilon$ is a sum of errors from several sources, then no matter what the probability distribution of the separate errors may be, their sum $\varepsilon$ will have a distribution that will tend more and more to the normal distribution as the number of components increase. Thus there is a tendency for errors that occur in many real situations to be normally distributed. It is reasonable to conclude that the voxels in EIT image data are normally distributed. Furthermore, if the experimental effects in EIT imaging are correctly specified, the error term in the GLM should be normally distributed.

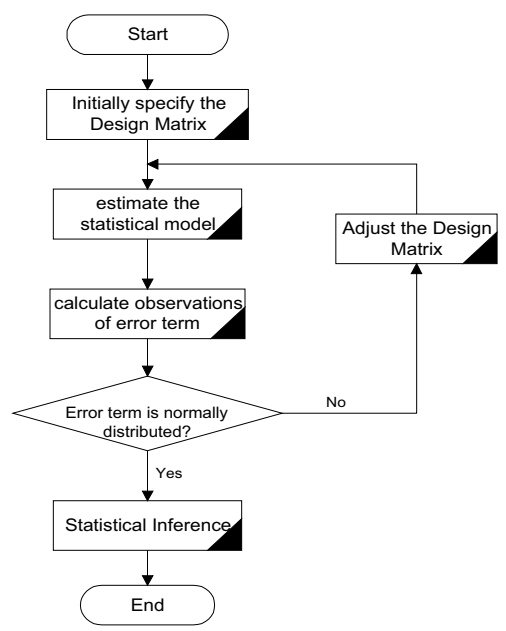

Figure 1: Scheme for the processing of EIT data
Based on previous analyses, a scheme is proposed for the processing of EIT data with SPM (see figure 1). In order to test the practicality of this scheme, some experiments have been carried out and more details are mentioned in the next section.

\subsection{Analysis of Simulated EIT Data in SPM}

EIT researchers usually use three kinds of data: simulated data, phantom data and clinical data. During the collection of clinical and phantom data, there are always some unpredictable factors that degrade the image quality. With the intention of making the data more controllable and to facilitate the evaluation of experiment results, simulated data was used in this study.

\subsubsection{Simulated Data Creation}

As mentioned previously, in brain EIT imaging, functional impedance changes, with a time-course of minutes, may be caused by cells swelling or blood volume and flow increase. In this experiment, we created some datasets to model the conductivity change caused by blood volume and flow increase. Noise included in clinical EIT imaging is modelled as well. Finally, three types of simulated datasets are generated. The following sections describe them in detail.

\section{- Type I - Noise-free Dataset}

This type of dataset aims to model an ideal situation: how the impedance changes according to time is precisely known and no noise is introduced.

The size of the simulated datasets was $200 \times 200 \times 200 \times 36(x, y, z, t)$, which means there are 36 sample points, at each sample point, a volume dataset with size $200 \times 200 \times 200$ is produced. The time interval between the first three sample points is one second, and then the time interval changed to one third second. Coordinates in these datasets are defined as: $x$ increases from left to right, $y$ increases from posterior to anterior, and $z$ increases from inferior to superior. $x, y$ and $z$ are all changed from -100 to 100 . The origin $(0,0,0)$ is defined as the centre of the brain.

To create the simulated datasets, the human brain is modelled as a simple sphere with constant impedance. The blood volume and flow increase region, which will be referred as the ROI (Region of Interest), is also defined as a sphere. This ROI is centred at the point $(-40,0,0)$, with radius of 20 . A function is used to express the impedance change ratio in the ROI:

$$
t_{-} \text {course }=1+\left(3 \times t-0.55 \times t^{2}+0.025 \times t^{3}\right) / 100
$$

As pointed out previously, blood volume and flow increase raises the local brain impedance by a few 
percent. The amplitude of this defined impedance change is about five percent.

EIT reconstruction algorithms usually include two steps: the solution of forward problem and the solution of inverse problem. In order to create the simulated data, we first calculated the boundary voltages by solving the forward problem. Then, the volume imaging dataset is reconstructed from these boundary voltages with TSVD (Truncated Singular Value Decomposition) reconstruction algorithm [1]. The flow chart in figure 2 illustrates the procedure to form the dataset.

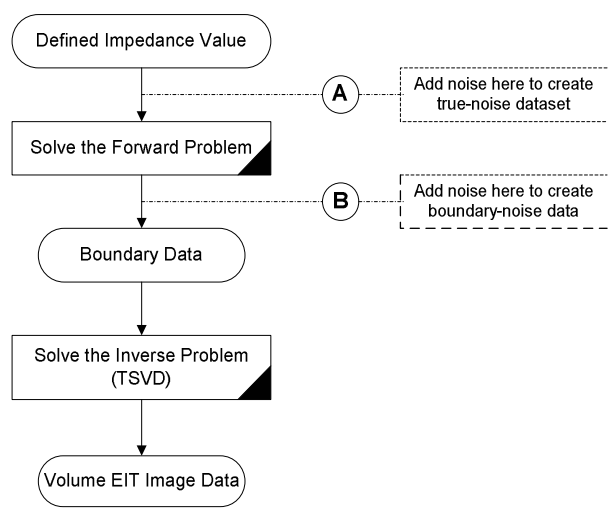

Figure 2: Simulated data creation procedure

\section{- $\quad$ Type II - True-noise dataset}

In clinical application, it is almost impossible to describe impedance change in the brain exactly. Usually, only a rough change tendency can be deduced based on certain principles. The second type of dataset used, termed the true-noise dataset, tries to simulate this situation by adding noise before the solution of forward problem (see point $\mathrm{A}$ in figure 2). The other steps in the creation of this type of dataset are same as those in the creation of noise-free dataset.

\section{- Type III - Boundary-noise dataset}

Beside the unpredictability of the impedance change value, another noise source in real measurement is that caused by imaging equipment. In order to imitate this environment, we produced the third type of dataset: boundary-noise dataset, which has a similar procedure as the creation of the second type dataset, but more noise is added after the solution of forward problem (see point B in figure 2).

Table 1: Overview of the Simulated Datasets

\begin{tabular}{|c|l|c|}
\hline Dataset No. & \multicolumn{1}{|c|}{ Noise Type } & $\begin{array}{c}\text { Noise } \\
\text { Amplitude }\end{array}$ \\
\hline Dataset 1 & Noise free & -- \\
\hline Dataset 2 & True noise & $10 \%$ \\
\hline Dataset 3 & True noise & $20 \%$ \\
\hline Dataset 4 & Boundary noise & $2 \%$ \\
\hline Dataset 5 & Boundary noise & $5 \%$ \\
\hline
\end{tabular}

By adjusting the noise ratio, different datasets can be produced. Table 1 shows some general information about the datasets used in the experiments.

\subsubsection{Model Selection and Spatial Normalisation}

The first step in using SPM to process image data is to decide which statistical models to adopt. Two statistical models are provided in SPM: one for PET/SPECT data, and the other for fMRI data. Generally, PET analysis is a little simpler than fMRI analysis. The reason is that, for PET, the observations (voxel values) are nearly independent (which means that the signal that generated the voxel value for one scan has more or less decayed to negligible levels by the time of next scan) for the relevant long time intervals. However, the time spacing between fMRI scans within a scan session is often very short; in this case the signal that generated one scan may still be presented at the time of the next.

In the simulated datasets, for a single voxel, the impedance value at each sample point is specified precisely. There is no interaction between values at different time points. The observations can be treated as independent. Therefore, PET statistical model is selected in the experiment.

After specification of statistical model, next steps in the processing are: spatially normalise the data, specify the Design Matrix and other parameters, and subsequently, the statistical model is estimated and statistical inference is carried out.

Spatial normalization aims to reduce artifactual variance components in the time-series which are generally induced by head movements of subject among a series of scans or shape differences among scans for different subjects. Because no movement or shape differences are contained in the simulated dataset, the spatial normalization step is omitted in the tests.

\subsubsection{Design Matrix and Parameters Specification}

Construction of the Design Matrix is a key point in the proposed scheme. In SPM, both PET and fMRI models provide different choices to form this matrix, which are classified according to the factors considered in the analysis, such as conditions, subjects, and sessions. In the estimated datasets, only one subject is considered and no experiment condition change is included. Therefore, the second design type in PET model - "Single-subject: covariates only" - is selected as a framework of the Design Matrix. The conductivity change and the scan time are defined as covariates in the initial Design Matrix. Other main parameters used in the experiments were set as table 2 . 
Table 2: Parameters used in the tests

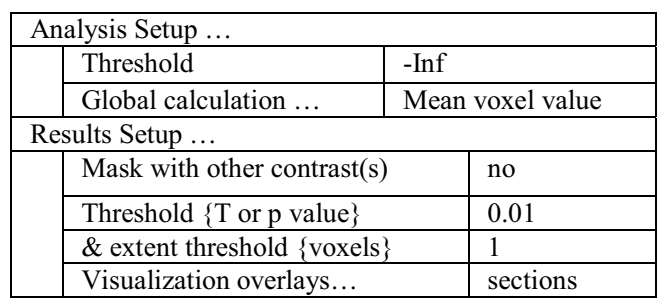

\subsubsection{Distribution of the error term}

According to equation (2), the error term in the General Linear Model can be expressed as:

$$
\underline{\varepsilon}=X-G \underline{\beta}
$$

After parameters estimation, errors of each voxel at every sample point, i.e. observations of the error term, can be calculated.

To test if a sample of data came from a population with a specific distribution, the Chi-square goodnessof-fit test is the most frequently used statistical method and it was adopted in our tests.

For every voxel, observations of the error term are sorted and divided into $\mathrm{K}$ groups. Each group ${ }^{i}$ has a maximum value $M A_{i}$ and a minimum value $M I_{i}$, and $M A_{i-1}=M I_{i}$. To test the null hypothesis $H_{0}$ that: $P_{i}=p_{i}$, the test statistic $T S$ in Chi-square test is:

$$
T S=\sum_{i=1}^{K} \frac{\left(N_{i}-e_{i}\right)^{2}}{e_{i}}
$$

Where $i=1, \ldots, K$ indexes the group

$N_{i}=n P_{i}$ is the observed frequency for group

$i, n$ is the sample size

$e_{i}=n p_{i}$ is the expected frequency for group

$i, n$ is the sample size..

Because we are testing whether the error term conforms to normal distribution, the expected frequency for group $i$ is:

$$
p_{i}=\int_{M I_{i}}^{M A_{i}} \frac{1}{\sqrt{2 \pi \sigma^{2}}} \exp \left(-\frac{(x-\mu)^{2}}{2 \sigma^{2}}\right) d x
$$

Where $\mu$ is the mean of the error distribution

$$
\sigma^{2} \text { is the variance of the error distribution }
$$

Mean ${ }^{\mu}$ and variance $\sigma^{2}$ of the error term can be obtained with Maximum Likelihood Estimation.

The degrees of freedom $d f$ of the Chi-square distribution, which approximates the test statistic $T S$, can be calculated with:

$$
d f=K-1
$$

Let $\chi_{K-1, \alpha}^{2}$ denote a Chi-square random variable having $(K-1)$ degrees of freedom will exceed this value with probability $\alpha$, then the approximated significance level $\alpha$ test of the null hypothesis $H_{0}$ against the alternative $H_{1}$ is as follows:

$$
\begin{array}{lc}
\text { Reject } H_{0} & \text { if } T S \geq \chi_{K-1, \alpha}^{2} \\
\text { Do not reject } H_{0} & \text { otherwise }
\end{array}
$$

\section{Results and Discussion}

\subsection{Datasets}

As described in section 3.2.1, five datasets were created to test the feasibility of using SPM to process EIT data. Figure 3 shows 2D displays of the timecourse transverse planes (where $\mathrm{z}=0$ ) of these datasets.

Observing the display for the first dataset, it is easy to find a global maximum, which is aunique local maximum in the dataset. The area around this maximum presents the position where the blood volume and flow increases, which is the ROI in this dataset. In the second and the fourth datasets, although not as easy as the first dataset, it is not difficult to find out the ROI. While in the third and the fifth datasets, especially in the fifth dataset, there are more than one local maximum. It would be hard to decide which one is the ROI if no information about how these datasets are created was available.

\subsection{Error Distribution}

Using the Design Matrix and parameters specified in section 3.2.3, the statistical model is estimated. The error terms are then calculated according to equation (5). In this section, the results of using Chi-square test to examine the distribution of error terms are given.

To make sure that there are at least 80 percent of the observed frequency $N_{i}$ exceed 5 in the test, the 36 observations of error terms are divided into 7 groups, so the degrees of freedom in the test is: $d f=7-1=6$. For the significance level $\alpha=0.005$, $\chi_{6,0.005}^{2}=18.55$.

Using the methods mentioned in section 3.2.4, the test statistic $T S$ is calculated for every voxel in each dataset, and then compared with $\chi_{6,0.005}^{2}$. Table 3 is an overview of the test results. The second column in table 3 is total number of voxels being statistically estimated in SPM. The third column is number of voxel whose $T S$ is greater than 18.55 and then whose 


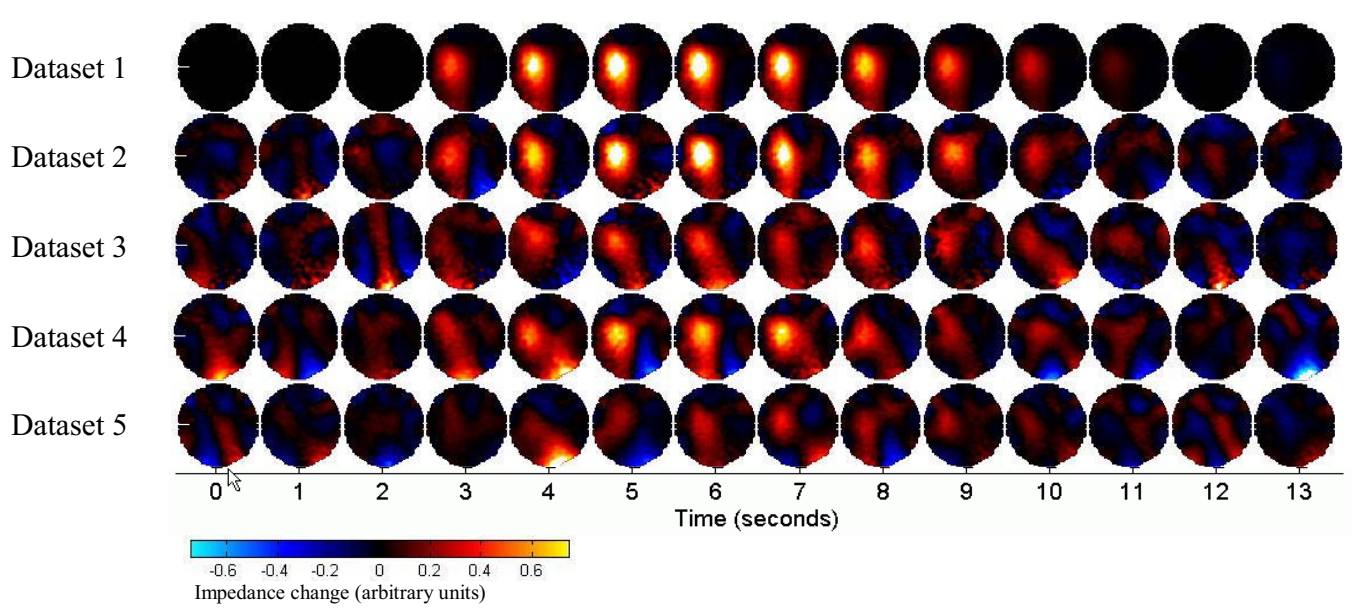

Figure 3: Time-course transverse planes $(\mathrm{z}=0)$ of the simulated datasets

normal distribution assumption is rejected. The last column displays the rejected ratio of this dataset. Test results show that all reject ratios are less than $2 \%$. Therefore, it is reasonable to draw a conclusion that the error terms are normally distributed in the specified GLM.

Table 3: Overview of Chi-square test results

\begin{tabular}{|c|c|c|c|}
\hline Dataset No. & $\begin{array}{c}\text { Total Voxel } \\
\text { Number }\end{array}$ & $\begin{array}{c}\text { Voxel Number } \\
\left(T S \geq \chi_{6,0.005}^{2}\right)\end{array}$ & Percentage \\
\hline Dataset 1 & 193046 & 2461 & $1.27 \%$ \\
\hline Dataset 2 & 229816 & 3976 & $1.73 \%$ \\
\hline Dataset 3 & 232771 & 3586 & $1.54 \%$ \\
\hline Dataset 4 & 210479 & 3389 & $1.61 \%$ \\
\hline Dataset 5 & 229586 & 3753 & $1.63 \%$ \\
\hline
\end{tabular}

According to the scheme defined in section 3.1, because the error terms are normally distributed, it is not necessary to refine the initial Design Matrix. Then the next step should be statistical inference.

\subsection{Statistical Inference Results}

SPM provides different methods to display the inference results. We adopted the approach to overlay the results on orthogonal sections of the processed image data. Figure 4 shows the statistical analysis results. In every result, except for dataset 1 , there is a closed zone highlighted by SPM, which seems to be covering the same region as defined for impedance variation. To test if it is true, we try to compare the centroid of the highlighted zone and the specified impedance variation area.

As mentioned in section 3.2.1, the centroid of the impedance change region is at point $(-40,0,0)$. The central voxel (rounded centroid position) for each highlighted zone is calculated and listed in the second column of table 4; the third column in this table presents the variation between the calculated central voxel and the centroid of the impedance change region. Dividing the variation by 200 (because $x, y$ and $z$ coordinate all change from -100 to 100 in the simulated data), we get the variation percentage. The last column of table 4 shows the biggest variation percentage among the three coordinates. Usually confidence level can be set as $95 \%$. In our tests, all the percentage variations (except dataset one) are less than $5 \%$. So the conclusion is drawn that SPM correctly located the centroid of the changed impedance region.

As mentioned before, the specified impedance change region in every simulated dataset is identical, however the areas marked by SPM varies between the datasets. We suggest this effect is caused by the smoothing effect of reconstruction processing of EIT data, which blurs the sharp change between the impedance variation region and other parts in the dataset. Voxels in this blurred area have same impedance change tendency as those voxels in the specified region, while their amplitude changes are smaller. With a specified probability threshold, some voxels in the blurred area are marked as statistically significant conforming to the defined function.

If there is some noise added in, some voxels in the blurred area, which have similar or smaller amplitude than the amplitude of noise, will not be included in the highlighted zone again, no matter how low the probability threshold is. In our experiments, the same probability threshold was used for every dataset. This made the area of marked zone change a lot: the higher amplitude of the noise, the smaller the highlighted zone. 


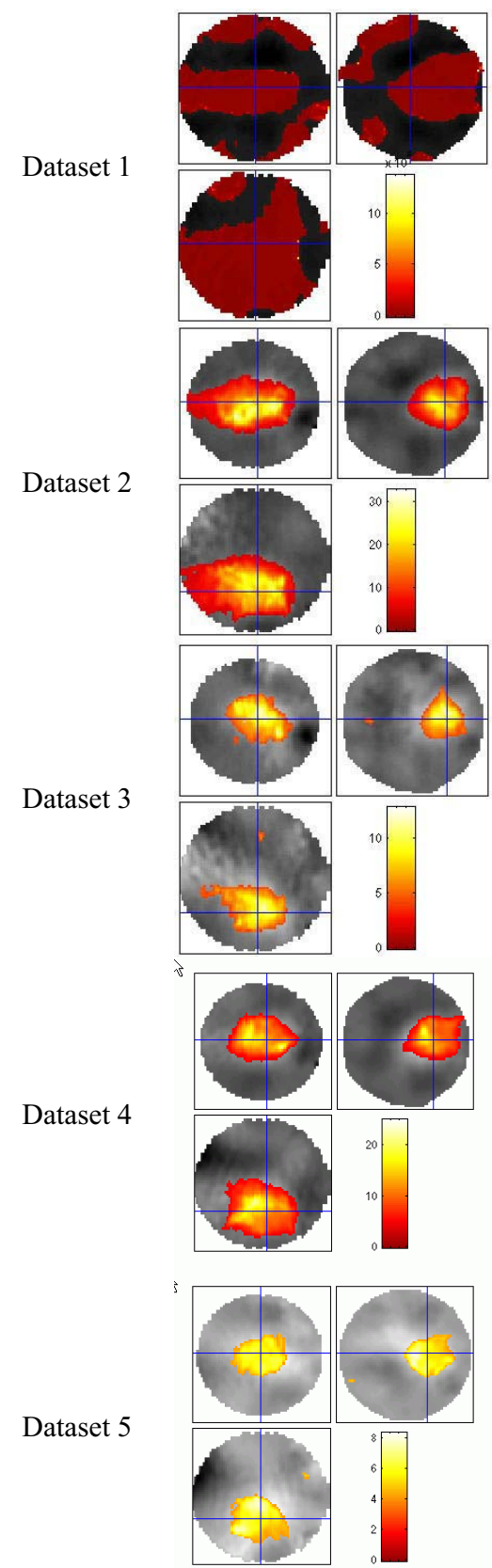

Figure 4: Statistical processing results set 1

Table 4: Analysis of centroids in results set 1

\begin{tabular}{|c|c|c|c|}
\hline $\begin{array}{c}\text { Dataset } \\
\text { No. }\end{array}$ & $\begin{array}{c}\text { Central } \\
\text { Voxel }\end{array}$ & Variation & Percent \\
\hline Dataset 1 & $(-23,-5,3)$ & $(17,15,3)$ & $8.5 \%$ \\
\hline Dataset 2 & $(-35,-7,-2)$ & $(5,-7,-2)$ & $3.5 \%$ \\
\hline Dataset 3 & $(-42,-5,-4)$ & $(-2,-5,-4)$ & $2.5 \%$ \\
\hline Dataset 4 & $(-37,-4,1)$ & $(3,-4,1)$ & $2 \%$ \\
\hline Dataset 5 & $(-41,-2,2)$ & $(-1,-2,2)$ & $1 \%$ \\
\hline
\end{tabular}

How to choose a suitable probability threshold for different datasets is an important topic that need to be investigated in future work. Besides the probability threshold, there is another threshold used in SPM, the voxel value threshold. By using the value threshold, all the voxels whose value is less than this threshold will not be included in the statistical calculation. In previous tests, the voxel value threshold is set as "-Inf" (see table 2), so all the voxels in the simulated dataset are included in the statistical analysis. If the previous explanation about the highlighted area change in the results is correct, adjusting the value threshold hopefully can enclose the highlighted region in the first dataset. Therefore, another investigation was conducted by specifying the value threshold to "ten percent of the maximum value". Figure 5 shows the results of this test.

Comparing the two result sets, it was found that the biggest change occurred in the first dataset, which now had a well defined area marked by SPM. Results for other datasets have not obviously changed. The certral voxel (rounded centroid position) of each highlighted region in the second set of tests is listed in table 5. Similar to the first set of experiment, the percentage variations for all centroids are still less than $5 \%$.

Dataset 1

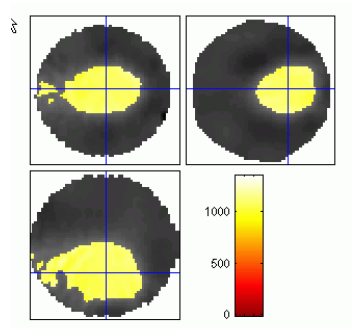

Dataset 2
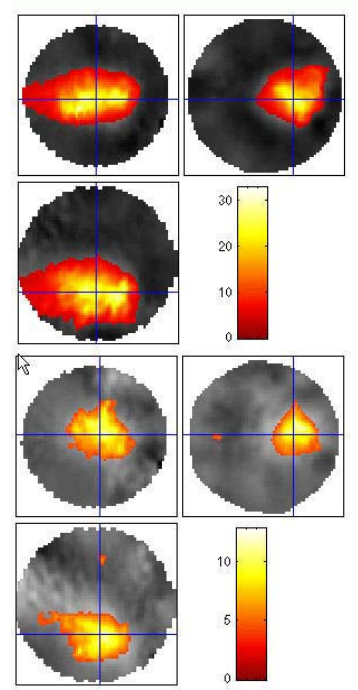
Dataset 4

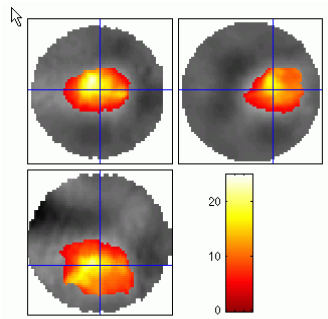

Dataset 5

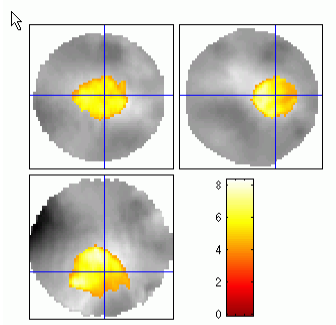

Figure 5: Statistical processing results set 2

Table 5: Analysis of centroids in results set 2

\begin{tabular}{|c|c|c|c|}
\hline $\begin{array}{c}\text { Dataset } \\
\text { No. }\end{array}$ & $\begin{array}{c}\text { Centrol } \\
\text { Voxel }\end{array}$ & Variation & Percent \\
\hline Dataset 1 & $(-37,9,1)$ & $(3,9,1)$ & $4.5 \%$ \\
\hline Dataset 2 & $(-39,-8,0)$ & $(1,-8,0)$ & $4 \%$ \\
\hline Dataset 3 & $(-42,-6,-3)$ & $(-2,-6,-3)$ & $3 \%$ \\
\hline Dataset 4 & $(-37,-3,2)$ & $(3,-3,2)$ & $1.5 \%$ \\
\hline Dataset 5 & $(-42,-3,0)$ & $(-2,-3,0)$ & $1.5 \%$ \\
\hline
\end{tabular}

\section{Conclusion}

EIT is a promising newcomer to functional brain imaging. Compared to more mature neuro-imaging methods, more work needs to be done on the analysis of EIT images. Our study suggests that it is appropriate to use the statistical methods provided by SPM to process EIT data. A scheme for the processing of EIT data with SPM has been proposed and experimental results suggest that it is reasonable to process EIT data with SPM according to this scheme as the centroid of the ROI, or the impedance variation region, in EIT image was correctly located to a given tolerance.

The presented results were based on simulated EIT data; future work will focus on clinical EIT data to further evaluate the efficacy of this scheme. Clinical EIT data is much more complex than simulated data. How to define the initial Design Matrix and adjust the Design Matrix properly is an important research topic.

\section{References}

[1] Avis N.J. and Barber D.C. (1994) Image Reconstruction Using Non-Adjacent Drive Configurations. Physiol. Meas.15 (Suppl. 2A): 153 - 160.

[2] Boone K., Lewis AM. and Holder D.S. (1994). "Imaging of cortical spreading depression by EIT:implications for localization of epileptic foci”. Physiological Measurement 15:189-198.

[3] Cox RW (1996). AFNI, software for analysis and visualization of functional magnetic resonance neuroimages. Comput Biomed Res 29:162-173.

[4] Duffy FH, B. P., Burchfiel JL (1981). "Significance probability mapping: an aid in the topographic analysis of brain electrical activity." Electriencephalogr Clin Neurophysiol 51(5): 455-462.

[5] Frackowiak R.S.J., Friston K.J., etc. editors. Human Brain Function (Chapter 1). Academic Press, 2nd edition, 2003.

[6] Friston KJ, Frith CD, Liddle PF, Frackowiak RSJ (1991): Comparing functional (PET) images: The assessment of significant change. J Cereb Blood Flow Metab 11:690-699.

[7] Friston KJ, Holmes AP, Worsley KJ, Poline JP, Frith CD, Frackowiak RSJ (1995) Statistical Parametric Maps in Functional Imaging: A General Linear Approach Human Brain Mapping 2:189-210

[8] Holder D.S. (1992). "Detection of cerebral ischaemia in the anaesthetized rat by impedance measurment with scalp electrodes: implications for non-invasive imaging of stroke by electrical impedance tomography." Clin. Phys. Physiol. Meas 13: 63-76.

[9] Lionheart W. R. B. (1997). Conformal uniqueness results in anisotropic electrical impedance imaging. Inverse Problems, 13:125-134.

[10] Liston AD. "Models and Image Reconstruction in Electrical Impedance Tomography of Human Brain Function”. PhD Thesis, Middlesex Univ.. 2004.

[11] Rao, A., A. Gibson, et al. (1997). "EIT imaging of electrically induced epileptic activity in anaesthetised rabbits." Med Bio Eng Comput 35(1): 3274-3284.

[12] Tidswell A.T., Gibson A., Bayford R.H. and Holder D.S. (2001) "Three Dimensional Electrical Impedance Tomography of Human Brain Activity". NeuroImage 13 (2): 283-294. 